\title{
Acute and Chronic Effects of Resistance Exercise on the Testosterone and Cortisol Responses in Obese Males: a Systematic Review
}

\author{
C. B. O'LEARY ${ }^{1}$, A. C. HACKNEY ${ }^{1}$ \\ ${ }^{1}$ Department of Exercise and Sport Science, University of North Carolina Chapel Hill, Chapel Hill, \\ NC, USA
}

Received July 30, 2013

Accepted January 17, 2014

On-line August 26, 2014

\begin{abstract}
Summary
The biosynthesis and metabolism of testosterone and cortisol are altered by the high levels of adipose tissue and the constant state of low-grade inflammation of obesity. Resistance exercise (REX) has become one of the main lifestyle interventions prescribed to obese individuals due to its ability to positively influence body composition and some biomarkers, such as cholesterol and insulin resistance. Yet, little research has been done in obese examining the effects of REx on the testosterone and blood cortisol responses, two integral hormones in both exercise and obesity. The obese testosterone response to REx and whether or not it is blunted compared to lean individuals remains elusive. Conflicting findings concerning the blood cortisol response have also been reported, likely due to variance in REx protocol and the level of obesity in the participants in studies. Comparatively, both of these hormones have been extremely well studied in untrained lean males, which could be used as a basis for future research in obese males. However, without this endocrinological information, it is unknown if the current acute REx prescriptions are appropriate for eliciting a favorable acute endocrinological response, and ultimately, a positive chronic adaptation in obese males.
\end{abstract}

\section{Key words}

Strength training $\bullet$ Endocrine $\bullet$ Hormones $\bullet$ Overweight

\section{Corresponding author}

A. C. Hackney, University of North Carolina, CB \# 8700 Fetzer Hall, Chapel Hill, NC 27599, USA. Fax: 919-962-0489. E-mail: ach@email.unc.edu

\section{Introduction}

The obesity epidemic continues to grow (Popkin and Doak 1998, Ogden et al. 2012), as individuals constantly intake more energy than they expend. Obesity is characterized as a chronic state of low-grade inflammation due to the high levels of adipose tissue and its constituents, which secrete adipokines, such as proinflammatory cytokines in the circulation (Johnson et al. 2012). This inflammatory state has negative systemic effects including: changes in metabolism, muscle morphology, endocrine, immunity, and cardiac function. The effects of these changes can lead to a decreased quality of life and increased risk of mortality and morbidity (Golbidi et al. 2012).

Two integral endocrine hormones greatly affected by obesity are testosterone and cortisol. Testosterone is produced by the testicles in males and is a powerful anabolic androgen hormone that stimulates muscle protein synthesis and intramuscular amino acid uptake, resulting in positive net protein balance and muscle hypertrophy (Vingren et al. 2010). Cortisol is a catabolic glucocorticoid hormone produced from the stimulation of the adrenal cortex by adrenocorticotropic hormone $(\mathrm{ACTH})$ in response to psychological and physiological stressors, including social pressures, extreme temperatures, and physical exercise. These hormones have significant effects on both protein metabolism and lipolysis, two metabolic pathways affected by obesity, but also essential in the management and treatment of obesity (McMurray and Hackney 2005).

PHYSIOLOGICAL RESEARCH • ISSN 0862-8408 (print) • ISSN 1802-9973 (online)

(c) 2014 Institute of Physiology v.v.i., Academy of Sciences of the Czech Republic, Prague, Czech Republic

Fax +420 241062 164, e-mail: physres@biomed.cas.cz, www.biomed.cas.cz/physiolres 
Exercise programs have long been used to combat the negative consequences of obesity by improving body composition, blood pressure, lipid profile, and glycemic control, along with restoring hormonal imbalances and preventing the development of obesity-related comorbidities (Strasser 2013). Yet, longterm exercise regimes are composed of multiple acute bouts of exercise, which makes the investigation of single exercise bouts important for understanding the acute physiological responses that ultimately lead to positive adaptations. Acute REx is a powerful stimulator of both the testosterone and blood cortisol responses in lean individuals (Kraemer and Ratamess 2005) and will be reviewed in regards to its effects on these hormonal responses.

This review attempts to present an overview of what is currently known on the topic of acute REx and its effect on testosterone and cortisol (unless otherwise noted, cortisol will refer to blood cortisol) in men and provide where greater knowledge is needed with the intent of providing guidance for future research endeavors. Organizationally, this review first provides a brief overview on how obesity affects the basic physiology of cortisol and testosterone. Next, it transitions into REx and the physiological benefits of this lifestyle intervention for obese individuals and the limited research pertaining to changes in hormonal profiles due to long-term REx. Finally, it concludes with research examining the acute effects of REx on each of these hormones, starting with a brief summary of lean individuals, followed by obese. There is considerable information given concerning the endocrinological response in lean individual as a point of reference for future research using an obese model. The discussion of the topic is delimited to studies using male subjects in order to avoid the complications presented by female sex hormones.

\section{Testosterone physiology and obesity}

Obesity is inversely related with testosterone concentrations in men, with stronger relationships being exhibited when using visceral adiposity measures compared to BMI (Mah and Wittert 2010). While these epidemiological studies report a relationship between decreased testosterone and obesity, both the cause and effect relationship and the exact pathophysiological mechanism for reduced testosterone in obese men are still partially unclear. Sex hormone-binding globulin (SHBG) levels are lowered due to elevated insulin concentrations, which could be due to increased adiposity and elevated cortisol levels in obese individuals (Huatanen 2000). However, testosterone concentrations were also found to be lower in obese men independent of reductions in SHBG, suggesting hypothalamic-pituitary level failure. The amplitude of luteinizing hormone pulses, but not the pulsitility of luteinizing hormone, is significantly attenuated in obese subjects (Wu et al. 2008). Obese males also have elevated circulating estradiol, which comes from the aromatization of circulating testosterone by adipose tissue and increases with additional fat mass (Zumoff et al. 1981). This aromatization actually leads to further fat accumulation and testosterone deficiency due to negative effects of estradiol on the gonadotrophinreleasing hormone release (Bagatell et al. 1994) (Fig. 1).

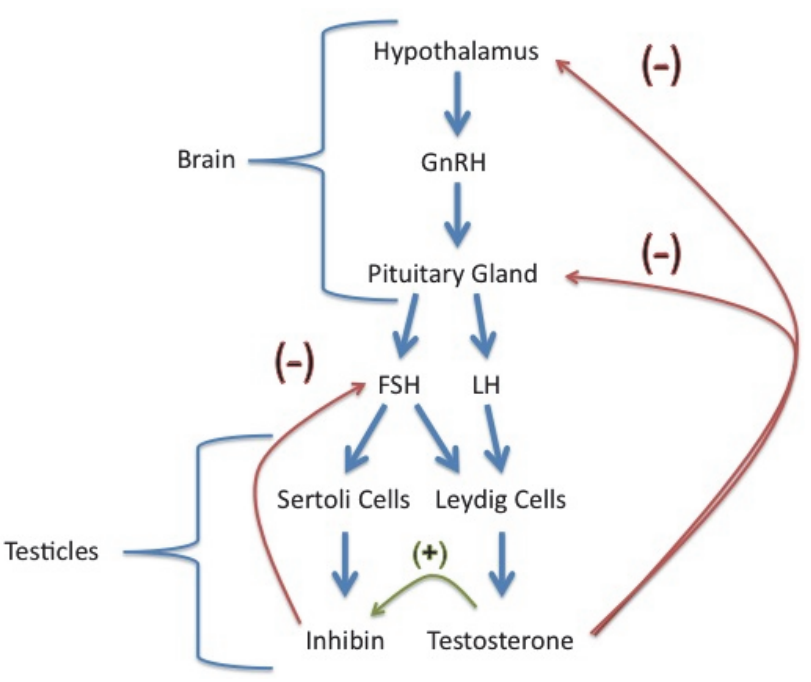

Fig. 1. Testosterone physiology overview.

Another possible link between obesity and testosterone dysfunction is a pathway involving leptin, a hormone released from adipose, which is elevated due to obesity. Leptin has been found to be the best hormonal predictor of androgen levels in obese men (Isidori et al. 1999). The exact physiological process between leptin and testosterone is unclear, but leptin receptors have been found in testicular tissue, providing a potential pathway for leptin to disrupt normal testosterone production. Other hormones, such as ghrelin, adiponectin, resistin, and endo-cannabinoids, have been studied in respect to obesity and testicular function, but their roles remain to be fully elucidated (Mah and Wittert 2010). Evidence exists that obesity and testosterone levels are linked. However, due to the complexity of the endocrine system 
and multiple pathways affecting testosterone, it is difficult to parse out the definite causes for the testosterone dysfunction evident in many obese males.

\section{Cortisol physiology and obesity}

Obesity appears to elevate cortisol due to either abnormal regulation of the HPA axis or possibly from peripheral modulations that are still only partially understood. When using urinary output, most studies have reported elevated cortisol outputs in obese individuals (Bjorntorp and Rosmond 2000). However, once corrections have been performed controlling for body surface area, cortisol output has been found to be elevated in some (Murphy 1968), but not other studies (Mussig et al. 2008). Studies examining serum cortisol have found increased concentrations (Bjorntorp and Rosmond 2000).

The chronic inflammation of obesity brings on a prolonged stress exposure (Johnson et al. 2012). Visceral fat releases pro-inflammatory cytokines such as interleukin-6, tumor necrosis factor- $\alpha$, and other adipocytokines into the circulation, which stimulates both the sympathetic nervous system and cortisol release (Black 2006). This prolonged stress exposure can ultimately burn out the HPA axis, causing a compensatory increase of the sympathetic nervous system. Catecholamines have been found to be proportionally greater in obese individuals, leading to increased activity of the HPA axis evidenced by amplified secretion of corticotropin-releasing hormone, ACTH, and cortisol (Bjorntrop and Rosmond 2000). Visceral adipose tissue, due to its large number of glucocorticoid receptors (GRs), is also particularly responsive to the actions of glucocorticoids, which further accentuates adipocyte differentiation and lipid deposition (Fried et al. 1993). However, high levels of cortisol are followed by a decrease in the number of GRs in adipose tissue, which attenuates lipolysis, further promoting the obese state (Matos et al. 2003).

Other possible mechanisms behind increased cortisol secretion in obese individuals are due to the enzymes located in peripheral tissues.

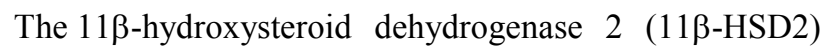
enzyme converts cortisol to the less active cortisone, and is found in the kidney, adipose tissue, muscle and liver (Seckl 1997). Due to the large amounts of excess adipose tissue containing this enzyme in obese individuals, there could be increased peripheral inactivation and metabolic clearance of cortisol. This would ultimately lead to an increased cortisol secretion by the HPA axis due to the peripheral dysfunction of clearance rates (Andrews and Walker 1999). Other research suggests that

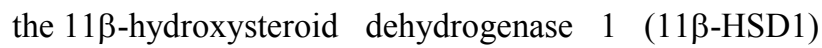
enzyme, which reactivates cortisone to active cortisol, also appears to be tissue-specifically enhanced in obesity (Rask et al. 2001). The dysregulation of peripheral cortisol enzymes appears to be one of the main causes of increased cortisol secretion of obesity. Obesity appears to have complex effects on cortisol and its biosynthesis pathways, which can lead to higher basal cortisol and a worsened obese state (Fig. 2).

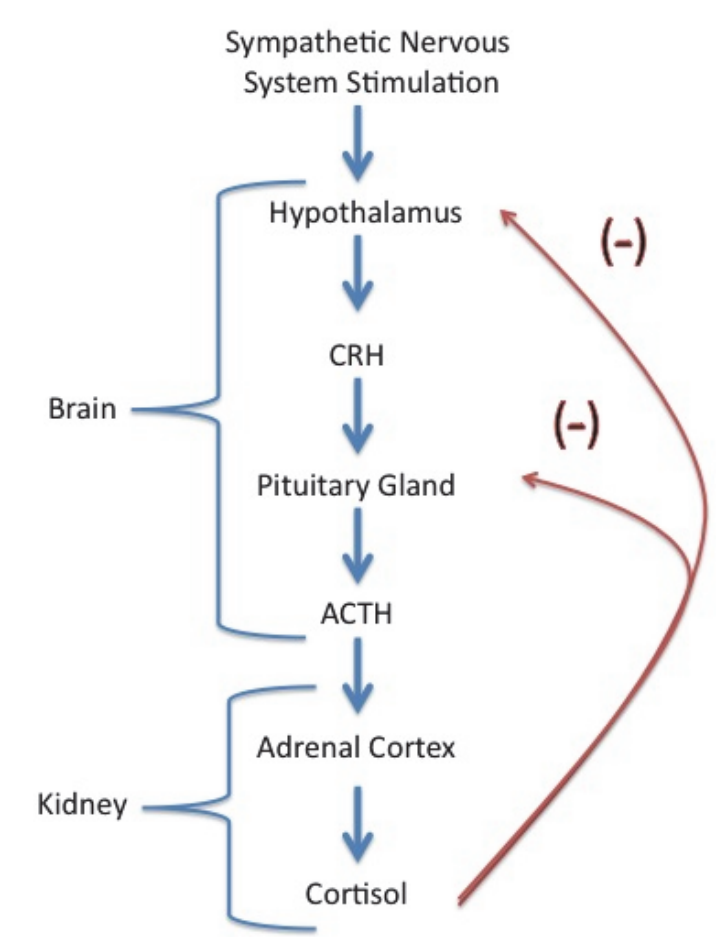

Fig. 2. Cortisol physiology overview.

\section{Resistance exercise and obesity}

REx, also known as strength training, dynamic strength training, or weight training, involves the voluntary activation of specific skeletal muscles against some form of external resistance. This external resistance is provided either by body mass, free weights, or a variety of exercise machines and implements done at variable intensities, volumes, and frequencies. Positive adaptations occur if a marginal overload stimulus, through increasing the intensity, volume or frequency of the lifting, is applied (Winett and Caripinelli 2001). If done correctly, REx is a safe, relatively simple activity, which does not necessarily involve using heavy weights, and takes minimal time (Carpinelli and Otto 1998). 
REx training studies involving adults show favorable changes for both metabolic and physical characteristics risk factors associated with osteoporosis, cardiovascular disease, cancer, and diabetes, along increases in muscle mass, strength, and integrity (Winett and Carpinelli 2001).

The increase in muscle mass, and subsequent elevation in resting metabolic rate, is one of the key adaptations of REx that benefits the obese (Ross et al. 1996). Since dieting alone usually causes decreases in both fat mass and lean body mass, the addition of REx to a dieting program may allow for the retention of lean body mass (Ross et al. 1996). The testosterone generated in response to REx plays a significant role in the preservation of lean body mass by increasing muscle protein synthesis and causing muscle hypertrophy (Vingren et al. 2010). The resulting microtrauma caused by REx also requires relatively large amounts of energy for the muscle remodeling process and may persist for up to six days after the exercise bout, further increasing energy expenditure and weight loss (Ahtiainen et al. 2011b).

Besides elevations in basal metabolic rate and lean body mass, resistance training also offers other benefits that lead to improved glycemic control and diabetes/metabolic syndrome prevention. Skeletal muscle glucose insulin-sensitive transporter type 4 content has been increased post-resistance training, possibly providing a mechanism behind the improved glycemic control (Gaster et al. 2002). There also seems to be decreases in glycosylated hemoglobin post-resistance training, indicating improved glycemic control and insulin sensitivity (Bacchi et al. 2012).

Many blood markers of obesity and obesityrelated comorbidities also improve after resistance training. Increases in high-density lipoproteins and decreases in low-density lipoproteins and triglycerides are exhibited post-resistance training (Kelley and Kelley 2009). There have also been documented cases of improvements in adiponectin and leptin, along with basal decreases in C-reactive protein, a common marker of inflammation. However, the effects of resistance training on other inflammatory cytokines and hormones remain unclear (Strasser et al. 2012).

\section{Hormone response to resistance training in obese}

The ability of resistance training to increase basal testosterone in lean adult males has been documented (Arazi et al. 2013). Yet, only one resistance training study has been completed using testosterone as an outcome measure in obese subjects that are not afflicted by any other metabolic disorders (Roberts et al. 2013). Thirty-six overweight/obese subjects were placed either in a control group $(n=8)$ or performed 12 weeks of resistance training $(n=24)$. The REx protocol followed American College of Sports Medicine guidelines by starting with high repetitions/low weight and progressing towards low repetitions/high weight over the course of the intervention. After the 12-week intervention, the REx group exhibited increases in fat free mass and decreases in fat mass, but no changes in basal total testosterone. There was actually a decrease in the basal concentration of free testosterone. This decrease in free testosterone could be due to the increased concentration of SHBG also reported post-intervention. A decrease in free testosterone could also come from an increase in androgen receptor (AR) content (Kraemer and Ratamass 2005), which would allow for more binding of free testosterone within the cell and facilitate hypertrophy. However, AR content was not measured by Roberts et al. (2013). Therefore at this time, it is still not understood how acute REx bouts should be composed to stimulate a long-term change in basal testosterone in obese individuals.

Basal cortisol levels were also examined by Roberts and colleagues (2013) after a resistance training intervention. Basal cortisol was found to decrease after the 12-week intervention. The authors cite the improvements in metabolic profile, especially the reductions in basal insulin levels, as the mechanism for the changes. Even though these obese individuals had normal fasting glucose and insulin levels before the intervention, resistance training still improved their insulin profile. No other measures of possible cortisol altering mechanisms, such as cortisol enzyme concentrations or catecholamine concentrations, were assessed in this study, making it unclear if the change in insulin was the only mechanism behind the decrease in basal cortisol.

\section{Acute testosterone response in lean}

Nine research studies have examined effects of acute REx the testosterone response using sedentary untrained lean subjects (Hansen et al. 2001, Tremblay et al. 2004, Baker et al. 2006, Bird et al. 2006, Yarrow et al. 2007, Cadore et al. 2008, 2009, Roberts et al. 2009, Ahtiainen et al. 2011a). Hansen and colleagues (2001) 
divided sixteen untrained sedentary men into either an arm group or arm + leg group. The arm group did four different bicep curl exercises [2 sets, 8-12 repetitions (reps) per set], while the arm + leg group did the same arm exercises and added 4 seated leg press exercises (2 sets, 8-12 reps per set). Blood samples were drawn pre-exercise after $15 \mathrm{~min}$ of supine rest, immediately post-exercise, and 15, 30, and 60 min into recovery. All of the exercise testing and training was done in the morning before 12:00 PM. The arm + leg group had increased testosterone immediately post-exercise, which returned to baseline within $15 \mathrm{~min}$ into recovery. The arm group did not exhibit any changes in testosterone at any of the measured time points, which may signify that a volume or intensity threshold is needed to induce a significant testosterone response. The inclusion of large muscle groups may also be necessary to induce an anabolic hormonal response.

Tremblay et al. (2004) evaluated the testosterone response as part of a study comparing the hormonal response of sedentary individuals to resistance-trained and aerobic-trained athletes. Seven sedentary subjects performed a REx bout, an aerobic exercise bout, and a control resting session. The REx bout was made up of three circuits of seven different exercises. Blood samples were drawn in a seated position pre-exercise after $30 \mathrm{~min}$ of rest and 1, 2, 3, and $4 \mathrm{~h}$ post-start of exercise. However, testing was done in the afternoon, which is not in agreement with the current literature concerning the appropriate time to evaluate the hormonal response due to the diurnal peak of testosterone (Baker et al. 2006, Cadore et al. 2008, 2009, Roberts et al. 2009). Another potential confounder in this study was the matching of the different exercise sessions according to the caloric expenditure measured via expired gases. This made the duration of the REx session vary between subjects, as the first 'post-exercise' blood sample could have occurred during the final portion of their REx instead of immediately post-exercise. Total testosterone was found to increase at the 'immediately' post-exercise time point ( $1 \mathrm{~h}$ post-start of exercise) before returning to and staying at basal levels for the other measured time points.

The testosterone response was examined as part of a study looking at the effect of nutritional interventions on acute biochemical responses to a single bout of REx in untrained men (Bird et al. 2006). This study utilized 32 sedentary untrained young men (18-29) placed equally into 4 nutrition groups (carbohydrate, protein, combined, and placebo) using a between-groups design. The subjects performed 3 sets of $8-10$ repetitions at $75 \%$ one repetition maximum (1RM) of 8 large muscle mass exercises, consuming $\sim 30 \mathrm{ml}$ of liquid between each set. Blood draws were performed pre-exercise, every $15 \mathrm{~min}$ during the exercise bout, immediately post-exercise, and 15 and $30 \mathrm{~min}$ into recovery. The exercise protocol was performed $4 \mathrm{~h}$ fasted between 15:00 and 17:00 PM to minimize the influence of diurnal variations of testosterone, with the times being held constant for each subject. The placebo group increased testosterone at both 15 and $30 \mathrm{~min}$ into the exercise session before returning to basal levels for the remaining blood draws. Unlike in other studies (Hansen et al. 2001, Tremblay et al. 2004), testosterone was not elevated immediately post-exercise, which reinforces the use of a design that includes the collection of blood samples during exercise necessary for categorizing the entire testosterone response.

Baker and colleagues (2006) examined the effects of aging on the testosterone response to acute REx. The researchers took 24 healthy sedentary untrained men in 3 different age groups (20-26, 38-53, 59-72 years old) and underwent an exercise protocol consisting of 6 exercises ( 3 sets of 10 reps at $80 \% 1 \mathrm{RM}$ ) covering the major muscle groups. Blood draws were completed preexercise, immediately post-exercise, and $15 \mathrm{~min}$ into recovery. Total and free testosterone increased immediately post-exercise, with a return to baseline within $15 \mathrm{~min}$ of cessation from exercise for all of the groups. The young, middle-aged, and older men all exhibited similar relative increases in testosterone; thus aging did not appear to attenuate the testosterone response to REx.

Yarrow and colleagues (2007) compared the anabolic responses after acute bouts of both traditional and eccentric REx protocols in sedentary untrained men. The traditional exercise bout consisted of 4 sets of 6 repetitions at $52.5 \% 1 \mathrm{RM}$ of both bench press and squat. The eccentric exercise was composed of 3 sets of 6 repetitions on both bench press and squat at $40 \% 1 \mathrm{RM}$ during the concentric phase and $100 \% 1 \mathrm{RM}$ during the eccentric phase. Blood samples were drawn pre-exercise after $10 \mathrm{~min}$ supine rest, immediately post-exercise, and $15,30,45$, and $60 \mathrm{~min}$ into recovery. Both protocols resulted in similar post-exercise anabolic hormone responses for all of the measured time points. Total testosterone did not change from baseline, except for a decrease at $60 \mathrm{~min}$ post-exercise. This study also measured the bioavailable (free and albumin-bound) portion of testosterone and found an increase immediately 
post-exercise, followed by a return to baseline, and ending with a decrease at $60 \mathrm{~min}$ post-exercise for both protocols. The authors suggest that concentric muscle action may be primarily responsible for the neuroendocrine responses of REx since the addition of overloaded eccentric muscle actions did not alter the hormonal response. The decreases in testosterone at the end of the protocol could be due to normal metabolic pathway biotransformation or through the binding of testosterone to muscle ARs, stimulating protein synthesis. Yet, this decrease in testosterone is not seen in other studies that analyzed blood more than one hour after completion of the protocol (Hansen et al 2001, Tremblay et al. 2004).

Two of the studies that examined testosterone were performed by the same research group using very similar study designs (Cadore et al. 2008, 2009). The only noticeable difference between the two studies was the use of serum (Cadore et al. 2008) instead of salivary (Cadore et al. 2009) measurements for the testosterone responses. Both studies compared the acute hormonal response to REx in strength-trained and sedentary men. The study design included four large muscle mass exercises. The exercise protocol was composed of 2 super sets, each consisting of 2 exercises ( 8 reps of each exercise at $75 \% 1 \mathrm{RM})$. Each superset was repeated 4 times for a total of 16 sets. Serum or saliva was taken pre-exercise and $10 \mathrm{~min}$ post-exercise, with the exercise protocol starting between 8:00 and 9:00 AM to account for any diurnal hormone variations. In the untrained group, there was a significant increase in serum free and total testosterone post-exercise (Cadore et al. 2008), but there were no increases in salivary testosterone (Cadore et al. 2009). The authors suggest that a high volume of acute REx might not be the correct protocol to use for untrained men due to the relatively high cortisol release, possibly inhibiting the secretion of testosterone from the testis (cortisol was increased post-exercise in both studies). However, the conflicting testosterone responses between the two studies may just be due to the sampling methodology. It is also hard to distinguish the full hormonal response using a study design with only two collection timepoints. Considering this, the immediately post-exercise hormonal responses found in the serum agree with previous research (Hansen et al. 2001, Tremblay et al. 2004).

Roberts and colleagues (2009) also examined the effects of aging on the acute testosterone response to REx using 11 young $(\sim 21$ years old) and 13 older $(\sim 67$ years old) healthy untrained, sedentary men. Both groups completed a REx protocol comprised of 3 sets of 10 repetitions at $80 \% 1 \mathrm{RM}$ of smith squats, bilateral leg press, and leg extension. Blood draws were completed pre-exercise, immediately post-exercise, and $24 \mathrm{~h}$ postexercise. This study only measured the free testosterone response, which has been found to parallel the total testosterone response (Tremblay et al. 2004). Age group differences were apparent, as basal free testosterone was greater in young compared to old men. Also, testosterone levels increased immediately post-exercise only in the young men, while the old men exhibited no change. The lack of change could be from not lifting enough weight due to their inexperience or fear of lifting heavy weights. It also could be due to their inability to produce testosterone because of their compromised gonadal system. This finding could be particularly applicable for hypothesizing what response might occur in obese individuals, as both aging (Harman et al. 2001) and obesity (Traish et al. 2009) can lower basal testosterone.

Another testosterone and aging related study was performed by Ahtiainen and colleagues (2011a), examining the effects of resistance training on resting testosterone and ARs in both young (25-30 years old) and old (60-65) sedentary untrained males. All subjects performed 5 sets of 10 reps at $100 \% 10 \mathrm{RM}$ of bilateral leg press for the experimental protocol. Blood samples were drawn pre-exercise, immediately post-exercise, and $15 \mathrm{~min}, 30 \mathrm{~min}$, and $48 \mathrm{~h}$ into recovery. Acute REx induced an increase in serum testosterone concentrations immediately post-exercise in both young and old individuals, with a return to near basal levels occurring by $15 \mathrm{~min}$ post-exercise. There was also no difference in the basal testosterone between the young and old groups. After reviewing the aging-related testosterone responses, it seems that having lower basal testosterone might lead to a reduced testosterone response after REx. However, in older individuals with normal testosterone, the hormonal response to REx seems to be similar to younger eugonadal men. This could be pertinent for obese individuals, as obesity, like aging, can induce hypogonadism, which could reduce the effects of REx on changes in testosterone.

REx appears to be able to properly stimulate an anabolic response in sedentary untrained males. To do so, an exercise protocol that is relatively high in both volume and intensity, along with utilizing large muscle groups and concentric muscle actions, is needed. Care must also be taken to standardize the time of day, along with the 
prandial conditions and sampling timepoints, in order to properly evaluate testosterone.

\section{Acute testosterone response in obese}

No study was found that examined the effects of acute REx on the testosterone response in obese individuals. This is in agreement with a previous review paper concerning hormone changes to acute exercise in obese individuals (Hansen et al. 2012).

\section{Acute cortisol response in lean}

Seven studies have examined the acute cortisol response to REx in lean sedentary males. Five of these studies were previously mentioned as they also examined testosterone (Hansen et al. 2001, Tremblay et al. 2004, Bird et al. 2006, Cadore et al. 2008, 2009). Hansen et al. (2001), who examined protocols using different amounts of muscle mass, found that the arm + leg group increased cortisol at $15 \mathrm{~min}$ into recovery but returned to baseline by $30 \mathrm{~min}$. The solely arm group actually exhibited an opposite response, as they had lower cortisol at 30 and 60 min into recovery compared to basal levels. Tremblay et al. (2004) examined different exercise types in trained and untrained individuals and found that REx increased cortisol both 'immediately' post-exercise and $2 \mathrm{~h}$ poststart of exercise, before returning to basal levels at $3 \mathrm{~h}$. The cortisol response lasted longer than in other studies, which could be due to the length and intensity of this specific exercise protocol and need for the body to increase cortisol to enhance glycogenolysis, gluconeogenesis, and protein catabolism to mobilize fuels for recovery and regeneration after exercise.

Cadore's research group found increases in cortisol immediately post-exercise in both serum (Cadore et al. 2008) and saliva (Cadore et al. 2009), but did not perform blood draws into recovery. Bird et al. (2006) also found that cortisol increased immediately after the commencement of the exercise bout and stayed elevated 30 min into recovery. The cortisol response appears to be specific to the exercise nutrition status and exercise protocol performed, as the intensity, lactate accumulation, total work performed, and rest period between sets help determine how much cortisol is released to stimulate glycogenolysis and gluconeogenesis (Kraemer et al. 1999).

Two studies examined solely the cortisol response to acute REx in untrained sedentary lean males without also examining the testosterone response (Kraemer et al. 1999, Fatouros et al. 2010). Kraemer and colleagues (1999) compared the acute response of stressrelated and fluid balance regulatory hormones to a single bout of REx in power lifters and untrained sedentary men (Kraemer et al. 1999). All subjects performed one set of bilateral leg press ( $80 \% 1 \mathrm{RM})$ until exhaustion, with no rest between repetitions. Blood draws occurred $30 \mathrm{~min}$ pre-exercise, immediately pre-exercise, immediately post-exercise, and $5 \mathrm{~min}$ post-exercise. There were no changes in cortisol at any time point in both groups and also no differences between the groups. Cortisol release appears to be dependent on the force of the muscle contraction, amount of muscle tissue stimulated, and rest period between the sets (Kraemer and Ratamass 2005), which was not great enough in this study to produce a response. The time course of a cortisol response may also occur later than 5 min into recovery, as other studies have shown changes from 15 (Hansen et al. 2001) to $60 \mathrm{~min}$ post-exercise (Tremblay et al. 2004). Low intensity exercise actually has a negative effect on cortisol release (Hansen et al. 2001), but the limited protocol of Kraemer et al. (1999) probably did not even allow for the body to make the necessary metabolic adjustments to reduce cortisol production.

The other study specifically examining the cortisol response was part of an investigation of a short bout of REx effects on catecholamines, cortisol, and cytokines in college-aged untrained sedentary males (Fatouros et al. 2010). Subjects completed 3 consecutive cycles of 10 exercises (70-75\% 1RM), with each cycle lasting about $10 \mathrm{~min}$. Subjects were given $30 \mathrm{~s}$ rest between exercises and 2 min of rest between each cycle. However, it is unclear how many repetitions were done for each exercise. Blood sampling was performed preexercise and at the end of each cycle but did not include any blood draws into recovery. The changes in serum cortisol during the exercise bout were not significant at any time point. This study only produced a moderate lactate response $(<10 \mathrm{mmol} / \mathrm{l})$, which may not have been large enough to elicit a cortisol response (Kraemer and Ratamass 2005). In general, cortisol has been found to either increase (Hansen et al. 2001, Tremblay et al. 2004, Cadore et al. 2008, 2009) or not change (Kraemer et al. 1999, Fatouros et al. 2010) after an acute bout of REx in sedentary untrained men. Findings seem dependent on the intensity of the exercise protocol or time of the blood draws, as increases in cortisol appear to occur postexercise. 


\section{Acute cortisol response in obese}

Only three research studies were found to have investigated the acute cortisol response using obese individuals (Chatzinikolaou et al. 2008, Mendham et al. 2011, Thomas et al. 2012). Chatzinikolaou and colleagues (2008) examined the cortisol response as part of an investigation evaluating the effects of acute REx on adipose tissue lipase in lean and obese men. This paper was only a rapid communication and not a full-length original research article, making most of the methodology and analysis brief in nature. The exercise protocol was composed of 3 cycles of 10 exercises selected to stress the major muscle groups. There were 10-12 repetitions per set at $70-75 \% 1 \mathrm{RM}$ with $30 \mathrm{~s}$ rest between sets and $2 \mathrm{~min}$ rests between cycles ( $\sim 30 \mathrm{~min}$ total). Blood and adipose tissue were sampled pre-exercise and at 5, 10, 20 and $30 \mathrm{~min}$ during exercise. Cortisol was elevated above baseline in the obese group at both 20 and $30 \mathrm{~min}$ into exercise. There was no change throughout the exercise bout for the lean individuals, which is in agreement with other studies using circuit training and lean untrained subjects (Fatouros et al. 2010). The obese group also had a larger absolute value of cortisol at the 30-min time point. This is the first study to suggest that the cortisol response may be altered due to obesity during REx. The altered response could be due to the reduced ability of obese individuals to oxide fat for fuel (Matos et al. 2003), forcing a greater cortisol response to generate fuel from glycogenolysis and gluconeogenesis. It could also be due to the relative difficulty and intensity of the exercise protocol for the untrained obese individuals. However, the lack of blood samples during recovery makes it difficult to determine the full magnitude of the augmented cortisol response in obese individuals.

Further research examining the cortisol response was done as part of a study investigating the acute REx-induced cytokine and leukocyte response to resistance and aerobic exercise (Mendham et al. 2011). Twelve sedentary untrained overweight male subjects were used in this study. This study failed to achieve recruit subjects that were solely obese, as the mean BMI was 29.4 (obese $=$ BMI $>30$ ). However, total body fat mass was $27.3 \pm 5.4 \%$ (mean \pm SEM) when evaluated by dual-energy X-ray absorptiometry, which is above the threshold for being considered obese obesity (25\% for males) (Okorodudu et al. 2010). The exercise protocol was conducted in the morning, but the individuals' drank a standardized protein sports drink supplement $2 \mathrm{~h}$ before exercise, possibly affecting the cortisol response. In terms of the actual exercise protocol, subjects performed 3 sets of 10 repetitions for 7 different exercises. Two different exercises intensities were compared on separate days, one done at $60 \%$ 1RM (low intensity) and the other performed at $80 \% 1 \mathrm{RM}$ (moderate intensity). Blood samples were drawn pre-exercise, immediately postexercise, and 3 and $24 \mathrm{~h}$ post-exercise. The moderate intensity protocol induced an increased cortisol response immediately post-exercise, with concentrations returning to pre-exercise values at $3 \mathrm{~h}$ post-exercise. Low intensity exercise did not stimulate a change in cortisol at any time point. There was also no lean control group, making it difficult to establish if the cortisol response was actually augmented. Yet, the lack of decrease in cortisol during the low intensity exercise could be viewed as an augmentation when compared to previous studies done in lean individuals, as low intensity REx can cause a decrease in cortisol during recovery (Hansen et al. 2001). Due to the heterogeneity of the subjects and lack of a control group, along with the possible effects of prior feeding, the results of this study must be cautiously interpreted for an obese population.

The most recent study examining the cortisol response in an obese sample was done as part of a study examining the effects of an acute REx protocol on the glucoregulatory and ghrelin responses (Thomas et al. 2012). Ten healthy untrained sedentary obese males and nine lean untrained sedentary controls participated in the study. However, this study split its obese population into two separate groups based upon their level of obesity as defined by the World Health Organization (WHO) (WHO1=30-35 BMI, WHO2/3=>35 BMI). This classifying was probably done so the researchers could report significant differences in their main outcome variables, but it is important to keep in mind that the level of obesity could greatly affect the hormonal response in future studies. All subjects performed the exercise tests between 6:30 and 11:00 AM to account for the circadian rhythms and diurnal peaks of hormone secretion. The subjects performed 3 sets of 10 repetitions at 85-95\% 10RM on 6 different exercises. Blood samples were collected pre-exercise after $20 \mathrm{~min}$ of quiet sitting, immediately post-exercise, and 50, 70, and $110 \mathrm{~min}$ into recovery. REx produced an increase in cortisol above preexercise values at all of the post-exercise time points in all three groups. When the cortisol response was evaluated using area under the curve data, the WHO2/3 group had less of an overall cortisol response compared 
to the WHO1 group (with no difference between lean control and $\mathrm{WHO} 2 / 3$ ). This lower cortisol response in the very obese was hypothesized to be due to chronically high cortisol concentrations and a blunted stress response (Thomas et al. 2012). However, there was no difference in basal cortisol between any of the groups. While there does seem to be some kind of alteration in the cortisol response due to obesity, this study does not fully elucidate the change. The conflicting results found in these studies makes future research necessary as to identify how obesity affects the cortisol response to REx.

\section{Conclusion}

After completing this review it is evident that substantial research has been done concerning the effects of acute exercise on testosterone and cortisol in lean individuals. Both testosterone and cortisol have been found to increase post-exercise, as testosterone increases are exhibited immediately post-exercise, while the cortisol response is slightly delayed into recovery depending on the exercise protocol. For these changes to occur, a protocol sufficient in both volume and intensity must be used to produce and secrete these hormones.

Yet, there are some gaps in the literature pertaining to obesity, REx, and subsequent cortisol and testosterone responses. The cortisol response appears to be dependent on the characteristics of the exercise protocol and the individual's level of obesity, which necessitates valid and reliable body composition measurements. The design must also take into account the possibility of the hormonal response being altered both during and after a bout of REx due to obesity. This makes blood draws before, during, immediately after, and into recovery essential for completely characterizing the response. Basal testosterone levels should also be considered, as eugonadal and hypogonadal obese individuals may respond differently to REx, even if the level of obesity is similar. Considering the lack of research for the acute effects of testosterone, future research should focus on implementing protocols that have elicited positive testosterone changes in lean individuals, but that are also safe for obese people. Future studies must work towards finding ideal resistance exercise protocols for obese individuals by comparing lean and obese individuals using the same REx protocol as to compare the response. However, it is still unclear whether obese individuals need to perform REx at similar intensities as lean individuals to stimulate these hormones. Once sufficient protocols have been developed, long-term training studies should then be done to examine if REx is appropriate for restoring any endocrinological dysfunctions in obese individuals. In general, REx seems to be a safe and an effective intervention that promotes positive metabolic and endocrinology changes in lean individuals. Further research must still be done on acute REx bouts using obese individuals before conclusions about the endocrinological response and proper exercise prescriptions can be made for obese individuals performing REx.

\section{Conflict of Interest}

There is no conflict of interest.

\section{References}

AHTIAINEN JP, HULMI JJ, KRAEMER WJ, LEHTI M, NYMAN K, SELANNE H, ALEN M, PAKARINEN A, KOMULAINEN J, KOVANEN V, MERO AA, HAKKINEN K: Heavy resistance exercise training and skeletal muscle androgen receptor expression in younger and older men. Steroids 76: 183-192, $2011 \mathrm{a}$.

AHTIAINEN JP, LEHTI M, HULMI JJ, KRAEMER WJ, ALEN M, NYMAN K, SELANNE H, PAKARINEN A, KOMULAINEN J, KOVANEN V, MERO AA, HAKKINEN K: Recovery after heavy resistance exercise and skeletal muscle androgen receptor and insulin-like growth factor-I isoform expression in strength trained men. J Strength Cond Res 25: 767-777, 2011 b.

ANDREWS RC, WALKER BR: Glucocorticoids and insulin resistance: Old hormones, new targets. Clin Sci (Lond) 96: 513-523, 1999.

ARAZI H, DAMIRCHI A, ASADI A: Age-related hormonal adaptations, muscle circumference and strength development with 8weeks moderate intensity resistance training. Ann Endocrinol (Paris) 74: 30-35, 2013. 
BACCHI E, NEGRI C, ZANOLIN ME, MILANESE C, FACCIOLI N, TROMBETTA M, ZOPPINI G, CEVESE A, BONADONNA RC, SCHENA F, BONORA E, LANZA M, MOGHETTI P: Metabolic effects of aerobic training and resistance training in type 2 diabetic subjects: a randomized controlled trial (the RAED2 study). Diabetes Care 35: 676-682, 2012.

BAGATELL CJ, DAHL KD, BREMNER WJ: The direct pituitary effect of testosterone to inhibit gonadotropin secretion in men is partially mediated by aromatization to estradiol. $J$ Androl 15: 15-21, 1994.

BAKER JR, BEMBEN MG, ANDERSON MA, BEMBEN DA: Effects of age on testosterone responses to resistance exercise and musculoskeletal variables in men. J Strength Cond Res 20: 874-881, 2006.

BIRD SP, TARPENNING KM, MARINO FE: Effects of liquid carbohydrate/essential amino acid ingestion on acute hormonal response during a single bout of resistance exercise in untrained men. Nutrition 22: 367-375, 2006.

BJORNTORP P, ROSMOND R: The metabolic syndrome--a neuroendocrine disorder? Br J Nutr 83 (Suppl 1): S49-S57, 2000.

BLACK PH: The inflammatory consequences of psychologic stress: Relationship to insulin resistance, obesity, atherosclerosis and diabetes mellitus, type II. Med Hypotheses 67: 879-891, 2006.

CADORE EL, LHULliER FL, BRENTANO MA, DA SILVA EM, AMBROSINI MB, SPINELLI R, SILVA RF, KRUEL LF: Hormonal responses to resistance exercise in long-term trained and untrained middle-aged men. J Strength Cond Res 22: 1617-1624, 2008.

CADORE EL, LHULLIER FL, ARIAS BRENTANO M, MARCZWSKI DA SILVA E, BUENO AMBROSINI M, SPINELLI R, FERRARI SILVA R, MARTINS KRUEL LF: Salivary hormonal responses to resistance exercise in trained and untrained middle-aged men. J Sports Med Phys Fitness 49: 301-307, 2009.

CARPINELLI R, OTTO R: Strength training: Single versus multiple sets. Sports Med 26: 73-84, 1998.

CHATZINIKOLAOU A, FATOUROS I, PETRIDOU A, JAMURTAS A, AVLONITI A, DOUROUDOS I, MASTORAKOS G, LAZAROPOULOU C, PAPASSOTIRIOU I, TOURNIS S, MITRAKOU A, MOUGIOS $\mathrm{V}$ : Adipose tissue lipolysis is upregulated in lean and obese men during acute resistance exercise. Diabetes Care 31: 1397-1399, 2008.

FATOUROS I, CHATZINIKOLAOU A, PALTOGLOU G, PETRIDOU A, AVLONITI A, JAMURTAS A, GOUSSETIS E, MITRAKOU A, MOUGIOS V, LAZAROPOULOU C, MARGELI A, PAPASSOTIRIOU I, MASTORAKOS G: Acute resistance exercise results in catecholaminergic rather than hypothalamic-pituitaryadrenal axis stimulation during exercise in young men. Stress 13: 461-468, 2010.

FRIED SK, RUSSELL CD, GRAUSO NL, BROLIN RE: Lipoprotein lipase regulation by insulin and glucocorticoid in subcutaneous and omental adipose tissues of obese women and men. J Clin Invest 92: 2191-2198, 1993.

GASTER M, VACH W, BECK-NIELSEN H, SCHRODER HD: GLUT4 expression at the plasma membrane is related to fibre volume in human skeletal muscle fibres. APMIS 110: 611-619, 2002.

GOLBIDI S, MESDAGHINIA A, LAHER I: Exercise in the metabolic syndrome. Oxid Med Cell Longev 2012: $349710,2012$.

HANSEN S, KVORNING T, KJAER M, SJOGAARD G: The effect of short-term strength training on human skeletal muscle: the importance of physiologically elevated hormone levels. Scand J Med Sci Sports 11: 347-354, 2001.

HANSEN D, MEEUSEN R, MULLENS A, DENDALE P: Effect of acute endurance and resistance exercise on endocrine hormones directly related to lipolysis and skeletal muscle protein synthesis in adult individuals with obesity. Sports Med 42: 415-431, 2012.

HARMAN SM, METTER EJ, TOBIN JD, PEARSON J, BLACKMAN MR; BALTIMORE LONGITUDINAL STUDY OF AGING: Longitudinal effects of aging on serum total and free testosterone levels in healthy men. Baltimore Longitudinal Study of Aging. J Clin Endocrinol Metab 86: 724-731, 2001.

HAUTANEN A: Synthesis and regulation of sex hormone-binding globulin in obesity. Int J Obes Relat Metab Disord 24 (Suppl 2): S64-S70, 2000.

ISIDORI AM, CAPRIO M, STROLLO F, MORETTI C, FRAJESE G, ISIDORI A, FABBRI A: Leptin and androgens in male obesity: evidence for leptin contribution to reduced androgen levels. J Clin Endocrinol Metab 84: 3673-3680, 1999.

JOHNSON AR, MILNER JJ, MAKOWSKI L: The inflammation highway: metabolism accelerates inflammatory traffic in obesity. Immunol Rev 249: 218-238, 2012. 
KELLEY GA, KELLEY KS: Impact of progressive resistance training on lipids and lipoproteins in adults: a metaanalysis of randomized controlled trials. Prev Med 48: 9-19, 2009.

KRAEMER WJ, RATAMESS NA: Hormonal responses and adaptations to resistance exercise and training. Sports Med 35: 339-361, 2005.

KRAEMER WJ, FLECK SJ, MARESH CM, RATAMESS NA, GORDON SE, GOETZ KL, HARMAN EA, FRYKMAN PN, VOLEK JS, MAZZETTI SA, FRY AC, MARCHITELLI LJ, PATTON JF: Acute hormonal responses to a single bout of heavy resistance exercise in trained power lifters and untrained men. Can J Appl Physiol 24: 524-537, 1999.

MAH PM, WITTERT GA: Obesity and testicular function. Mol Cell Endocrinol 316: 180-186, 2010.

MATOS FAG, MOREIRA RO, GUEDES EP: Neuroendocrine aspects of metabolic syndrome (in Portugese). Arq Bras Endocrinol Metabol 47: 410-420, 2003.

MCMURRAY RG, HACKNEY AC: Interactions of metabolic hormones, adipose tissue and exercise. Sports Med 35: 393-412, 2005.

MENDHAM AE, DONGES CE, LIBERTS EA, DUFFIELD R: Effects of mode and intensity on the acute exerciseinduced IL-6 and CRP responses in a sedentary, overweight population. Eur J Appl Physiol 111: 1035-1045, 2011.

MUSSIG K, REMER T, HAUPT A, GALLWITZ B, FRITSCHE A, HARING HU, MASER-GLUTH C: 11 betahydroxysteroid dehydrogenase 2 activity is elevated in severe obesity and negatively associated with insulin sensitivity. Obesity (Silver Spring) 16: 1256-1260, 2008.

OGDEN CL, CARROLL MD, KIT BK, FLEGAL KM: Prevalence of obesity in the United States, 2009-2010. NCHS Data Brief (82): 1-8, 2012.

OKORODUDU DO, JUMEAN MF, MONTORI VM, ROMERO-CORRAL A, SOMERS VK, ERWIN PJ, LOPEZJIMENEZ F: Diagnostic performance of body mass index to identify obesity as defined by body adiposity: a systematic review and meta-analysis. Int J Obes (Lond) 34: 791-799, 2010.

POPKIN BM, DOAK CM: The obesity epidemic is a worldwide phenomenon. Nutr Rev 56: 106-114, 1998.

RASK E, OLSSON T, SODERBERG S, ANDREW R, LIVINGSTONE DE, JOHNSON O, WALKER BR: Tissuespecific dysregulation of cortisol metabolism in human obesity. J Clin Endocrinol Metab 86: 1418-1421, 2001.

ROBERTS MD, DALBO VJ, HASSELL SE, KERKSICK CM: The expression of androgen-regulated genes before and after a resistance exercise bout in younger and older men. J Strength Cond Res 23: 1060-1067, 2009.

ROBERTS CK, CROYMANS DM, AZIZ N, BUTCH AW, LEE CC: Resistance training increases SHBG in overweight/obese, young men. Metabolism 62: 725-733, 2013.

ROSS R, RISSANEN J, PEDWELL H, CLIFFORD J, SHRAGGE P: Influence of diet and exercise on skeletal muscle and visceral adipose tissue in men. J Appl Physiol 81: 2445-2455, 1996.

SECKL JR: Glucocorticoids, feto-placental 11 beta-hydroxysteroid dehydrogenase type 2, and the early life origins of adult disease. Steroids 62: 89-94, 1997.

STRASSER B: Physical activity in obesity and metabolic syndrome. Ann NY Acad Sci 1281: 141-159, 2013.

STRASSER B, ARVANDI M, SIEBERT U: Resistance training, visceral obesity and inflammatory response: a review of the evidence. Obes Rev 13: 578-591, 2012.

THOMAS GA, KRAEMER WJ, COMSTOCK BA, DUNN-LEWIS C, VOLEK JS, DENEGAR CR, MARESH CM: Effects of resistance exercise and obesity level on ghrelin and cortisol in men. Metabolism 61: 860-868, 2012.

TRAISH AM, FEELEY RJ, GUAY A: Mechanisms of obesity and related pathologies: Androgen deficiency and endothelial dysfunction may be the link between obesity and erectile dysfunction. FEBS J 276: 5755-5767, 2009.

TREMBLAY MS, COPELAND JL, VAN HELDER W: Effect of training status and exercise mode on endogenous steroid hormones in men. J Appl Physiol 96: 531-539, 2004.

VINGREN JL, KRAEMER WJ, HATFIELD DL, ANDERSON JM, VOLEK JS, RATAMESS NA, THOMAS GA, HO JY, FRAGALA MS, MARESH CM: Effect of resistance exercise on muscle steroidogenesis. J Appl Physiol 105: 1754-1760, 2008. 
VINGREN JL, KRAEMER WJ, RATAMESS NA, ANDERSON JM, VOLEK JS, MARESH CM: Testosterone physiology in resistance exercise and training: The up-stream regulatory elements. Sports Med 40: 1037-1053, 2010.

WU FC, TAJAR A, PYE SR, SILMAN AJ, FINN JD, O'NEILL TW, BARTFAI G, CASANUEVA F, FORTI G, GIWERCMAN A, HUHTANIEMI IT, KULA K, PUNAB M, BOONEN S, VANDERSCHUEREN D; EUROPEAN MALE AGING STUDY GROUP: Hypothalamic-pituitary-testicular axis disruptions in older men are differentially linked to age and modifiable risk factors: the European Male Aging Study. J Clin Endocrinol Metab 93: 2737-2745, 2008.

YARROW JF, BORSA PA, BORST SE, SITREN HS, STEVENS BR, WHITE LJ: Neuroendocrine responses to an acute bout of eccentric-enhanced resistance exercise. Med Sci Sports Exerc 39: 941-947, 2007.

ZUMOFF B, STRAIN GW, KREAM J, O'CONNOR J, LEVIN J, FUKUSHIMA DK: Obese young men have elevated plasma estrogen levels but obese premenopausal women do not. Metabolism 30: 1011-1014, 1981. 